\title{
Does visceral adiposity index signify early metabolic risk in children and adolescents?: Association with insulin resistance, adipokines, and subclinical inflammation
}

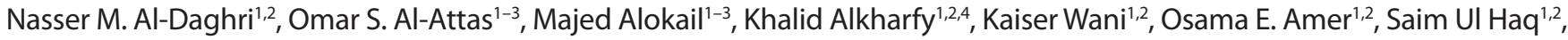 \\ Shakilur Rahman ${ }^{1,2}$, Abdullah M. Alnaami ${ }^{1,2}$, Sarantis Livadas ${ }^{5}$, Anastasios Kollias ${ }^{5}$, Paris Charalampidis ${ }^{6}$ and Shaun Sabico ${ }^{1,2}$
}

BACKGROUND: Visceral adiposity index (VAl) is a novel gender-specific index based on waist circumference (WC), BMI, and lipid parameters. Although VAI does not actually estimate visceral adiposity, it accurately reflects visceral fat function and insulin resistance. This index has not been studied in children thus far. This study aims to fill this gap.

METHODS: In a cohort of Saudi children and adolescents, anthropometric measurements and metabolic/hormonal profile were obtained.

RESULTS: A total of 543 subjects, 292 of whom were boys, were included (mean age: $11.9 \pm 3.3$ y; BMl: $19.8 \pm 5.6 \mathrm{~kg} / \mathrm{m}^{2}$ ). In all subjects, VAl was inferior to BMI and WC regarding its correlations with adiponectin, leptin, insulin resistance (homeostasis model of assessment-insulin resistance (HOMA-IR)), C-reactive protein (CRP) level, and systolic blood pressure, but it exhibited a stronger association with glucose in boys $(r=0.23 ; P<0.01)$. In stepwise multivariate analyses, only BMI was consistent as an independent predictor of adiponectin, leptin, HOMA-IR, and CRP. VAI was the only index independently associated with glucose.

CONCLUSION: Although VAl is related to glucose in children, it seems to be inferior to BMl in terms of association with insulin resistance, adipokines, and subclinical inflammation. Until specific studies can be performed in children, VAl should be extrapolated with caution in this age range.

W orldwide prevalence of childhood obesity has increased at alarming rates: about one-third of the young population is within the range of overweight/obesity (1-5). This is especially worrying because obesity is associated with subclinical inflammation and confers an increased cardiovascular risk $(6,7)$. More specifically, adipose tissue-derived factors that comprise the family of adipokines are considered to play a crucial role in promoting inflammation, insulin resistance, and atherosclerosis (8).

In everyday clinical practice, $\mathrm{BMI}$ has been established as the main anthropometric index for the assessment of childhood overweight/obesity and international cutoff values exist (9). However, BMI reflects overall fat and, in this context, waist circumference (WC) has been adopted to provide information about abdominal fat and for prediction of cardiovascular risk $(10,11)$.

Recently, research interest has been focused on the function of visceral fat. It has been demonstrated that subcutaneous and visceral (both omental and mesenteric) fat mass represents adipose tissue fractions with diverse actions in terms of insulin sensitivity, lipolytic activity, and adipokine production (11). More specifically, visceral fat is associated with the production of adipokines that play a key role in the determination of insulin sensitivity and in the initiation and maintenance of subclinical inflammation (8). In this context, visceral adiposity index (VAI) - a novel index derived from anthropometric (BMI and WC) and lipid parameters-has been shown to be associated with visceral adipose tissue and, moreover, with adipokine levels, mainly adiponectin, insulin resistance, and glucose disturbances in adults (12-15). In addition, this index seems to have predictive value in terms of an increased risk for cardiovascular events (13) and has served as a marker of insulin resistance in several different nosologic entities (16-21). Furthermore, our group recently reported, for the first time, the strong relationship of VAI with adipose tissue secretion and with functional glycemic disorders (22).

VAI is based on WC, BMI, and lipid parameters as follows: males: $\{[\mathrm{WC} / 39.68+(1.88 \times \mathrm{BMI})] \times(\mathrm{TG} / 1.03) \times(1.31 /$ HDL $)\}$; females: $\{[\mathrm{WC} / 36.58+(1.89 \times \mathrm{BMI})] \times(\mathrm{TG} / 0.81) \times$ $(1.52 / \mathrm{HDL})\}$. Here, TG stands for triglyceride level and HDL implies the high-density lipoprotein content. This index seems to reflect the severity of insulin resistance and exhibits a strong association with both the rate of peripheral glucose utilization during the euglycemic-hyperinsulinemic clamp-currently the gold standard of insulin resistance calculation-and the visceral adipose tissue measured with magnetic resonance imaging (12). However, to the best of our knowledge, there are no data concerning the value of VAI in children and adolescents. 
The aim of this study was to examine the association of VAI with insulin resistance, adipokine profile, and subclinical inflammation, compared with BMI and WC, in a large sample of children and adolescents.

\section{RESULTS}

From the initially screened children, 542 subjects satisfied the inclusion criteria and were included in the analysis. Their characteristics according to gender are presented in Table 1. Briefly, girls exhibited higher BMI, VAI, homeostasis model of assessment-insulin resistance (HOMA-IR), adiponectin, leptin, and resistin values, whereas tumor necrosis factor (TNF) $\alpha$ level was lower (Table 1). According to BMI status, overweight and obese children, compared with normal BMI children, demonstrated an adverse metabolic profile (Table 2). Moreover, VAI was increased across BMI categories in parallel with cardiometabolic characteristics (Table 2).

In bivariate analyses, BMI (mainly) and WC demonstrated the strongest associations with HOMA-IR, adiponectin, leptin, C-reactive protein (CRP), and systolic blood pressure (range of coefficients: 0.11-0.64) compared with VAI (range of coefficients: 0.008-0.28) and waist-hip ratio (range of coefficients: 0.001-0.25; Table 3). In particular, in the total sample, compared with VAI, both BMI and WC were more strongly associated with HOMA-IR $(r=0.27,0.43$, and 0.39 , respectively; $P<0.05$ for both BMI and WC, with $z$-statistic vs. that of VAI), leptin $(r=0.25,0.56$, and 0.46 , respectively; $P<0.01$ for both BMI and WC, with $z$-statistic vs. that of VAI), adiponectin

Table 1. Characteristics of the study population (mean $\pm \mathrm{SE}$ )

\begin{tabular}{|c|c|c|}
\hline Variable & Boys $(n=292)$ & Girls $(n=251)$ \\
\hline Age (y) & $11.9 \pm 0.19$ & $11.9 \pm 0.21$ \\
\hline $\mathrm{BMI}\left(\mathrm{kg} / \mathrm{m}^{2}\right)$ & $19.2 \pm 0.32$ & $20.5 \pm 0.35^{*}$ \\
\hline Overweight/obesity (\%) & $13 / 13$ & $17 / 14$ \\
\hline WC $(\mathrm{cm})$ & $69.8 \pm 1.38$ & $72.7 \pm 1.36$ \\
\hline VAI & $1.37 \pm 0.07$ & $2.17 \pm 0.11^{*}$ \\
\hline Glucose (mmol/l) & $5.01 \pm 0.04$ & $4.98 \pm 0.04$ \\
\hline Insulin ( $\mu \mathrm{U} / \mathrm{ml})$ & $12.9 \pm 1.95$ & $15 \pm 2.16^{*}$ \\
\hline HOMA-IR & $2.92 \pm 0.43$ & $3.30 \pm 0.46^{*}$ \\
\hline Systolic BP (mmHg) & $102.7 \pm 0.75$ & $103 \pm 0.92$ \\
\hline Diastolic BP (mmHg) & $67.1 \pm 0.55$ & $67.7 \pm 0.03$ \\
\hline LDL-cholesterol (mmol/l) & $2.79 \pm 0.04$ & $2.83 \pm 0.04$ \\
\hline HDL-cholesterol (mmol/l) & $0.98 \pm 0.02$ & $0.97 \pm 0.02$ \\
\hline Triglycerides (mmol/l) & $0.94 \pm 0.03$ & $0.97 \pm 0.03$ \\
\hline Adiponectin $(\mu \mathrm{g} / \mathrm{ml})$ & $19.5 \pm 0.73$ & $22.3 \pm 0.76^{*}$ \\
\hline Leptin (ng/ml) & $18.2 \pm 0.31$ & $30.5 \pm 0.39^{*}$ \\
\hline TNF- $\alpha(p g / m l)$ & $7.74 \pm 0.25$ & $6.65 \pm 0.28^{*}$ \\
\hline Resistin (ng/ml) & $16.4 \pm 0.53$ & $18.8 \pm 0.66^{*}$ \\
\hline CRP (mg/dl) & $0.29 \pm 0.04$ & $0.81 \pm 0.05$ \\
\hline \multicolumn{3}{|c|}{$\begin{array}{l}\text { BP, blood pressure; CRP, C-reactive protein; HDL, high-density lipoprotein; HOMA-IR, } \\
\text { homeostasis model of assessment-insulin resistance; LDL, low-density lipoprotein; } \\
\text { TNF, tumor necrosis factor; VAl, visceral adiposity index; WC, waist circumference. }\end{array}$} \\
\hline${ }^{*} P<0.01$ vs. boys. & & \\
\hline
\end{tabular}

( $r=-0.02,-0.25$, and -0.11 , respectively; $P<0.01$ only for BMI vs. VAI), and CRP $(r=0.08,0.26$, and 0.17 , respectively; $P<0.01$ for BMI vs. VAI). On the other hand, VAI tended to be superior only in terms of its association with glucose in boys (Table 3) (correlation coefficients: VAI-glucose $r=0.23$, BMI-glucose $r=0.15$ ( $z$-statistic: $0.96 ; P=0.17$ vs. $r$ of VAI-glucose), WCglucose $r=0.17$ ( $z$-statistic: $0.72 ; P=0.23$ vs. $r$ of VAI-glucose).

In stepwise multivariate analyses, BMI was consistently retained as independent predictor of adiponectin, leptin, HOMA-IR, and CRP, whereas glucose levels were predicted solely by VAI (Table 4 ). Female gender was also independently associated with higher adiponectin and leptin values (Table 4). The highest variance inflation factor was 1.22 (tolerance: 0.82 ) in these regression models, which ensures the lack of collinearity. It should be mentioned that the independent variables explained only a small amount of variance of the dependent variables.

\section{DISCUSSION}

The main findings of this study are that among all obesity indexes, BMI was superior in terms of the relationship with adipokines and cardiometabolic risk factors, but VAI was independently associated with fasting glucose levels.

Recent studies have demonstrated an increasing prevalence of childhood obesity (1-5), a fact with significant implications for public health because childhood obesity has an adverse impact on adult morbidity and mortality (6). Our results are

Table 2. Characteristics of the study population according to BMI status

\begin{tabular}{|c|c|c|c|}
\hline Variable & $\begin{array}{l}\text { Normal } \\
(n=389)\end{array}$ & $\begin{array}{l}\text { Overweight } \\
\quad(n=81)\end{array}$ & Obese $(n=73)$ \\
\hline Age (y) & $11.7 \pm 0.17$ & $12.9 \pm 0.30$ & $11.6 \pm 0.36^{*}$ \\
\hline $\mathrm{BMI}\left(\mathrm{kg} / \mathrm{m}^{2}\right)$ & $17.1 \pm 0.15$ & $24.1 \pm 0.30$ & $29.5 \pm 0.30^{* *}$ \\
\hline$W C(\mathrm{~cm})$ & $67.8 \pm 1.18$ & $79.4 \pm 2.1$ & $79.9 \pm 2.2^{* *}$ \\
\hline VAI & $1.69 \pm 0.08$ & $1.84 \pm 0.14$ & $1.90 \pm 0.19^{*}$ \\
\hline Glucose $(\mathrm{mmol} / \mathrm{l})$ & $4.95 \pm 0.03$ & $5.11 \pm 0.07$ & $5.08 \pm 0.09^{*}$ \\
\hline Insulin $(\mu \mathrm{U} / \mathrm{ml})$ & $12.3 \pm 1.61$ & $22.7 \pm 5.67$ & $13.5 \pm 2.22^{* *}$ \\
\hline HOMA-IR & $2.69 \pm 0.34$ & $5.18 \pm 1.27$ & $3.05 \pm 0.45^{* *}$ \\
\hline Systolic BP (mmHg) & $101.2 \pm 0.70$ & $106 \pm 1.3$ & $108.1 \pm 1.5^{* *}$ \\
\hline Diastolic BP $(\mathrm{mmHg})$ & $66.6 \pm 0.51$ & $68.7 \pm 0.93$ & $70 \pm 0.91^{* *}$ \\
\hline LDL-cholesterol (mmol/l) & $2.76 \pm 0.03$ & $2.95 \pm 0.08$ & $2.93 \pm 0.09^{*}$ \\
\hline HDL-cholesterol (mmol/l) & $1 \pm 0.02$ & $0.97 \pm 0.04$ & $0.88 \pm 0.03^{*}$ \\
\hline Triglycerides (mmol/l) & $0.93 \pm 0.03$ & $1 \pm 0.04$ & $1.04 \pm 0.05^{* *}$ \\
\hline Adiponectin $(\mu \mathrm{g} / \mathrm{ml})$ & $22.4 \pm 0.63$ & $15.9 \pm 1.35$ & $17.8 \pm 1.15^{* *}$ \\
\hline Leptin (ng/ml) & $17.1 \pm 2.8$ & $46.5 \pm 8.8$ & $34.8 \pm 4.9^{* *}$ \\
\hline TNF- $\alpha(p g / m l)$ & $7.14 \pm 0.22$ & $7.61 \pm 0.50$ & $7.37 \pm 0.51$ \\
\hline Resistin (ng/ml) & $16.9 \pm 0.50$ & $18 \pm 0.99$ & $20.2 \pm 1.19^{* *}$ \\
\hline $\mathrm{CRP}(\mathrm{mg} / \mathrm{dl})$ & $0.26 \pm 0.04$ & $0.37 \pm 0.07$ & $2.14 \pm 1.56^{* *}$ \\
\hline
\end{tabular}

BP, blood pressure; CRP, C-reactive protein; HDL, high-density lipoprotein; HOMA-IR, homeostasis model of assessment-insulin resistance; LDL, low-density lipoprotein; TNF, tumor necrosis factor; VAl, visceral adiposity index; WC, waist circumference.

${ }^{*} P<0.05,{ }^{* *} P<0.01$ for comparison among the three groups. 
Table 3. Bivariate associations between anthropometric indexes and metabolic characteristics

\begin{tabular}{|c|c|c|c|c|c|c|}
\hline Variable & VAl & BMI & WC & WHR & $\begin{array}{l}\text { HOMA- } \\
\text { IR }\end{array}$ & CRP \\
\hline \multicolumn{7}{|l|}{ Boys } \\
\hline Age & $0.35^{* *}$ & $0.36^{* *}$ & $0.48^{* *}$ & 0.08 & $0.30 * *$ & 0.09 \\
\hline VAI & & $0.26^{* *}$ & $0.57^{* *}$ & $0.24^{* *}$ & $0.28^{* *}$ & 0.12 \\
\hline BMI & $0.26^{* *}$ & & $0.45^{* *}$ & $0.14^{* *}$ & $0.37^{* *}$ & $0.23^{* *}$ \\
\hline WC & $0.57^{* *}$ & $0.45^{* *}$ & & $0.57^{* *}$ & $0.37^{*}$ & $0.17^{* *}$ \\
\hline WHR & $0.24^{* *}$ & $0.14^{*}$ & $0.57^{* *}$ & & $0.25^{* *}$ & -0.003 \\
\hline HOMA-IR & $0.28^{* *}$ & $0.37^{* *}$ & $0.37^{* *}$ & $0.25^{* *}$ & & $0.16^{*}$ \\
\hline Glucose & $0.23^{* *}$ & $0.15^{*}$ & $0.17^{* *}$ & 0.04 & $0.42^{* *}$ & 0.02 \\
\hline CRP & 0.12 & $0.23^{* *}$ & $0.17^{* *}$ & -0.003 & $0.16^{*}$ & \\
\hline Adiponectin & -0.08 & $-0.25^{* *}$ & -0.11 & 0.09 & -0.11 & -0.05 \\
\hline Leptin & $0.17^{* *}$ & $0.46^{* *}$ & $0.40^{* *}$ & $0.17^{* *}$ & $0.44^{* *}$ & $0.32^{* *}$ \\
\hline Resistin & -0.01 & $0.14^{*}$ & -0.09 & -0.11 & 0.08 & 0.06 \\
\hline TNF- $\alpha$ & 0.002 & $0.12^{*}$ & 0.04 & 0.003 & 0.11 & $0.15^{*}$ \\
\hline Systolic BP & $0.17^{* *}$ & $0.37^{* *}$ & $0.26^{* *}$ & 0.08 & $0.21^{* *}$ & $0.22^{* *}$ \\
\hline Diastolic BP & 0.12 & $0.24^{*}$ & $0.20^{* *}$ & 0.04 & $0.17^{*}$ & 0.14 \\
\hline $\begin{array}{l}\text { LDL- } \\
\text { cholesterol }\end{array}$ & 0.03 & 0.04 & -0.09 & -0.09 & 0.004 & 0.03 \\
\hline \multicolumn{7}{|l|}{ Girls } \\
\hline Age & $0.13^{*}$ & $0.53^{* *}$ & $0.53^{* *}$ & -0.10 & $0.34^{* *}$ & 0.10 \\
\hline VAI & & $0.18^{* *}$ & $0.40^{* *}$ & $0.23^{* *}$ & $0.16^{*}$ & $<0.001$ \\
\hline BMI & $0.18^{* *}$ & & $0.61^{* *}$ & 0.04 & $0.45^{* *}$ & $0.29 * *$ \\
\hline WC & $0.40^{* *}$ & $0.61^{* *}$ & & $0.54^{* *}$ & $0.41^{* *}$ & $0.18^{*}$ \\
\hline WHR & $0.23^{* *}$ & 0.04 & $0.54^{* *}$ & & 008 & 0.001 \\
\hline HOMA-IR & $0.16^{*}$ & $0.45^{* *}$ & $0.41^{* *}$ & 0.08 & & 0.05 \\
\hline Glucose & $0.13^{*}$ & $0.17^{* *}$ & 0.11 & -0.03 & $0.33^{* *}$ & 0.10 \\
\hline CRP & -0.008 & $0.29^{* *}$ & $0.18^{*}$ & 0.001 & 0.05 & \\
\hline Adiponectin & -0.07 & $-0.32^{* *}$ & -0.11 & 0.04 & -0.06 & -0.11 \\
\hline Leptin & $0.15^{*}$ & $0.64^{* *}$ & $0.55^{* *}$ & 0.06 & $0.47^{* *}$ & $0.22^{* *}$ \\
\hline Resistin & 0.09 & $0.26^{*}$ & 0.08 & $-0.18^{* *}$ & $0.15^{*}$ & 0.11 \\
\hline TNF- $\alpha$ & $0.14^{*}$ & -0.05 & 0.07 & 0.09 & -0.07 & 0.10 \\
\hline Systolic BP & 0.04 & $0.32^{* *}$ & $0.25^{* *}$ & $-0.16^{*}$ & $0.20^{* *}$ & 0.05 \\
\hline Diastolic BP & -0.04 & $0.25^{* *}$ & $0.19^{* *}$ & -0.13 & $0.19^{*}$ & 0.01 \\
\hline $\begin{array}{l}\text { LDL- } \\
\text { cholesterol }\end{array}$ & 0.08 & 0.09 & -0.03 & -0.11 & -0.09 & 0.08 \\
\hline
\end{tabular}

BP, blood pressure; CRP, C-reactive protein; HDL, high-density lipoprotein; HOMA-IR, homeostasis model of assessment-insulin resistance; LDL, low-density lipoprotein; TNF, tumor necrosis factor; VAl, visceral adiposity index; WC, waist circumference; WHR, waist-hip ratio.

${ }^{*} P<0.05 .{ }^{*} P<0.01$.

in accordance with previous reports in Saudi Arabia showing increased rates of childhood obesity despite regional variations (5). It should be mentioned, however, that the classification of overweight/obese children is based on accepted cutoff values of BMI derived from an international representative population (9). In this study, obese and overweight children, compared with normal BMI children, had an adverse metabolic profile (lipids, blood pressure, HOMA-IR, adipokines, and CRP). These findings, although expected in line with the published literature (16), are particularly reassuring for the clinical application of international BMI cutoff points in young Saudi populations for the identification of high-risk subjects.

This is the first study to examine the potential extrapolation of VAI in children and adolescents. This index has been derived and validated in adult populations subjected to magnetic resonance imaging and euglycemic-hyperinsulinemic clamp studies (12-15). Similar studies would be required for the direct calculation of this index in children; however, these are difficult to be performed in large young populations. In our study, the examined obesity measures showed consistent associations with age, blood pressure, adipokines, HOMA-IR, and CRP; however, there was a trend for superiority (stronger correlation coefficients and independent predictive value in multivariate analyses) for BMI (mainly) and WC relative to VAI and waist-hip ratio. VAI is independently associated with glucose values, a finding that has been reported from our group in a recent study in adults (22); however, this finding cannot establish its predictive value concerning the future emergence of impaired glucose tolerance and/or diabetes. On the other hand, BMI - an index of overall adiposity - was strongly associated with leptin values, which in turn demonstrated the strongest correlations with insulin resistance and subclinical inflammation.

The role and the difference in visceral adipose tissue, compared with subcutaneous fat, in the development of insulin resistance and metabolic syndrome has been well recognized in adults (23). However, children and adolescents should not be considered as "small adults" when considering adipose tissue because different pathophysiological cues are encountered at different age groups (24-26). Considering the above as a whole, it is not surprising that a previous study demonstrated that, contrary to the situation in adults, visceral adipose tissue and inflammation markers were not correlated with insulin sensitivity in children (27). Moreover, another study showed that in prepubertal children, leptin emerged as a genderindependent discrimination marker of adiposity degree and as a useful, gender-associated predictor of systemic insulin resistance (28). These findings raise questions concerning the assessment and the role of visceral adiposity in children in contrast with the established data in adults. It is worthy to note however, that parent-offspring transmission of adipocytokines and metabolic traits is highly heritable in the cohort (Arabs) used in the study (29) and that although BMI, rather than VAI, can influence insulin resistance better in children, the genetic factor still plays a major role in assessing cardiometabolic risk.

In conclusion, overall adiposity in children as assessed by BMI can better explain adipokine status, metabolic parameters, insulin resistance, and subclinical inflammation compared with visceral obesity as reflected by the VAI. This is in contrast with reports in adults and needs verification. Accordingly, more studies should be performed in children to elucidate the usefulness of VAI in everyday clinical practice. 
Table 4. Stepwise multivariate regression analyses (only significant $\beta$ coefficients \pm SEs are reported)

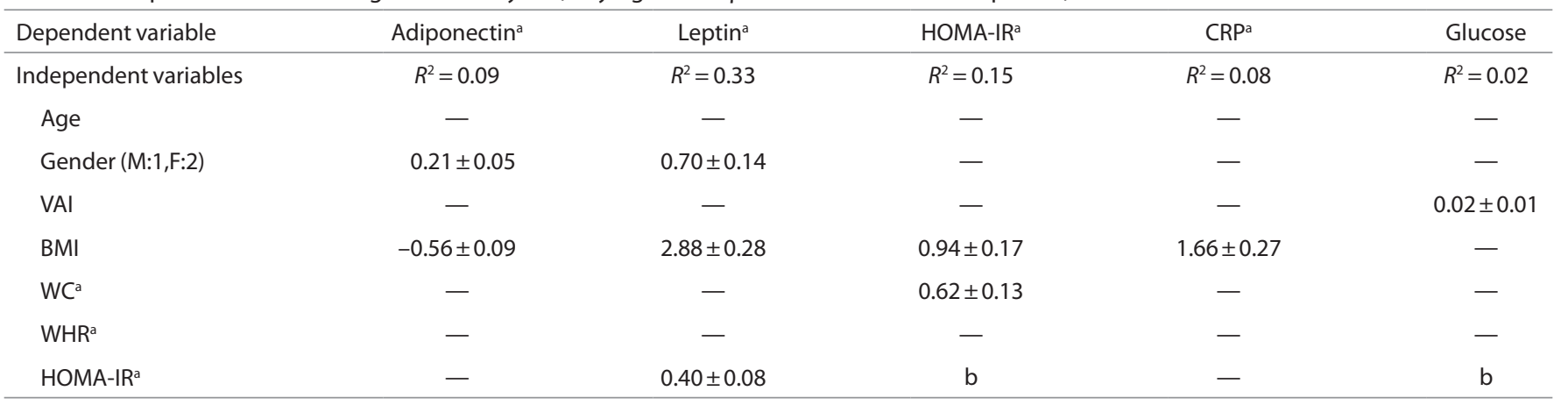

CRP, C-reactive protein; F, female; HOMA-IR, homeostasis model of assessment-insulin resistance; M, male; VAl, visceral adiposity index; WC, waist circumference; WHR, waist-hip ratio. aHOMA-IR was not included as independent variable. ' Logarithmically transformed.

\section{METHODS}

In this cross-sectional study, a total of 619 apparently healthy Saudi boys and girls aged $4-17 y$ were initially enrolled from 4 different Primary Health Care Centers in different areas within Riyadh city, Saudi Arabia, during the period March-December 2010. Subjects with chronic conditions such as asthma, type 1 diabetes mellitus, and hypertension; history of cardiac, kidney, or liver disease; use of medications known to affect body weight (such as steroids); and psychiatric conditions were excluded from the study. Subjects were assigned to the primary care center most convenient for them for the collection of anthropometric data and blood extraction. Physical examination was carried out by the attending physician who ensured that the participants met the inclusion and exclusion criteria. Weight and height were recorded to the nearest $0.2 \mathrm{~kg}$ and $0.5 \mathrm{~cm}$, respectively, using an appropriate international standard scale (Digital Pearson Scale; ADAM Equipment, Danbury CT). Additionally, waist and hip circumferences were measured using a standardized measuring tape in $\mathrm{cm}$, and the waist-hip ratio was estimated. Furthermore, BMI was estimated for each subject (weight/height ${ }^{2}$ ) as $\mathrm{kg} / \mathrm{m}^{2}$ and the Cole's criteria were used for the definition of overweight or obesity status (9); furthermore, child-appropriate indexes for BMI have been used (19). Blood pressure was measured using an appropriate mercury sphygmomanometer. Blood pressure was measured twice at 5-min intervals, and the mean of the two readings was recorded.

Blood was withdrawn by a nurse after an overnight fast $(>10 \mathrm{~h})$ on the same day that the anthropometric information was gathered, and serums were kept in the biobank of the Biomarkers Research Program at King Saud University. Serum insulin, leptin, adiponectin, TNF- $\alpha$, and resistin were quantified using multiplex assay kits that use fluorescent microbead technology, allowing simultaneous quantification of several target proteins in a single serum sample of $50-100 \mathrm{ml}$. These included premixed and fully customized panels that use the LuminexH xMAPH Technology platform (Luminexcorp, TX). For parameters measured using the multiplex assay, the intraassay variation was $1.4-7.9 \%$ and the interassay variation was $0.21 \%$. Minimum detectable concentrations were as follows: insulin: $50.9 \mathrm{pg} /$ $\mathrm{ml}$; leptin: $85.4 \mathrm{pg} / \mathrm{ml}$; adiponectin: $145.4 \mathrm{pg} / \mathrm{ml}$; resistin: $6.7 \mathrm{pg} / \mathrm{ml}$; TNF- $\alpha$ : $0.14 \mathrm{pg} / \mathrm{ml}$; and plasminogen activator inhibitor- $1: 1.3 \mathrm{pg} / \mathrm{ml}$. High-sensitivity CRP was determined using enzyme-linked immunosorbent assays (Immunodiagnoztik AG, Bensheim, Germany), with intra-assay variability of $5.5-6.0 \%$ and interassay variation of $11.6-$ $13.8 \%$. All fasting samples fell within the detection range.

Based on fasting values, HOMA-IR, an index of insulin resistance, was calculated as follows: HOMA-IR $=\left(\mathrm{I}_{0}{ }^{\prime} \times \mathrm{G}_{0}{ }^{\prime}\right) / 22.5$, where $\mathrm{I}_{0}{ }^{\prime}$ is the fasting insulin $(\mu \mathrm{U} / \mathrm{ml})$ and $\mathrm{G}_{0}{ }^{\prime}(\mathrm{mmol} / \mathrm{l})$ is the fasting glucose. VAI score was calculated as described (12) using the following gender-specific equations: for males: $\{[\mathrm{WC} / 39.68+(1.88 \times \mathrm{BMI})] \times(\mathrm{TG} / 1.03)$ $\times(1.31 / \mathrm{HDL})\}$; for females: $\{[\mathrm{WC} / 36.58+(1.89 \times \mathrm{BMI})] \times(\mathrm{TG} / 0.81)$ $\times(1.52 / \mathrm{HDL})\}$, where TG levels are expressed in $\mathrm{mmol} / \mathrm{l}$ and HDLcholesterol levels are expressed in $\mathrm{mmol} / \mathrm{l}$.

Ethical approval was obtained from the Ethics Committee of the College of Science Research Center, King Saud University, Riyadh, Saudi
Arabia. Written informed consents for parents and assent for children and/or adolescents were obtained before inclusion into the study.

\section{Statistical Analysis}

Statistical analysis was performed using the Statistical Package for Social Sciences software (SPSS release 17.0; SPSS, Chicago, IL). Results are presented as mean \pm SE. The Kolmogorov-Smirnov statistic was used to test continuous variables for normality. Spearman or Pearson correlation coefficients $(r)$ were determined for the assessment of the relationship between obesity indexes, adipokines, HOMA-IR, and CRP. The difference between correlation coefficients was tested with Meng's $z$-statistic method. Mann-Whitney or unpaired $t$-test was applied to compare variables between boys and girls. Kruskal-Wallis or ANOVA test was performed for comparisons among the three groups. Moreover, the $\chi^{2}$ test was applied for comparison of percentages. Stepwise multivariate regression analysis was performed to define independent predictors of adipokines, glucose, HOMA-IR, and CRP. Independent variables included gender, age, and all the obesity indexes. Variables that did not have a normal distribution were logarithmically transformed before entering into the model (WC, waist-hip ratio, adiponectin, leptin, HOMA-IR, and CRP). Tolerance and variance inflation factor were used for the assessment of potential multicollinearity among the variables retained in the final regression models. A probability value of $P<0.05$ was considered statistically significant.

\section{STATEMENT OF FINANCIAL SUPPORT}

The study is funded by the Deanship of Scientific Research at King Saud University (RGP-VPP-313).

\section{Disclosure: The authors have nothing to disclose.}

\section{REFERENCES}

1. Wang Y, Lobstein T. Worldwide trends in childhood overweight and obesity. Int J Pediatr Obes 2006;1:11-25.

2. Karnik S, Kanekar A. Childhood obesity: a global public health crisis. Int J Prev Med 2012;3:1-7.

3. Sekhobo JP, Edmunds LS, Reynolds DK, Dalenius K, Sharma A. Trends in prevalence of obesity and overweight among children enrolled in the New York State WIC program, 2002-2007. Public Health Rep 2010;125:218-24.

4. Kollias A, Skliros E, Stergiou GS, Leotsakos N, Saridi M, Garifallos D. Obesity and associated cardiovascular risk factors among schoolchildren in Greece: a cross-sectional study and review of the literature. J Pediatr Endocrinol Metab 2011;24:929-38.

5. El Mouzan MI, Al Herbish AS, Al Salloum AA, Al Omar AA, Qurachi MM. Regional variation in prevalence of overweight and obesity in Saudi children and adolescents. Saudi J Gastroenterol 2012;18:129-32.

6. Park MH, Falconer C, Viner RM, Kinra S. The impact of childhood obesity on morbidity and mortality in adulthood: a systematic review. Obes Rev 2012;13:985-1000.

7. Lloyd LJ, Langley-Evans SC, McMullen S. Childhood obesity and risk of the adult metabolic syndrome: a systematic review. Int J Obes (Lond) 2012;36:1-11. 
8. Libby P, Okamoto Y, Rocha VZ, Folco E. Inflammation in atherosclerosis: transition from theory to practice. Circ J 2010;74:213-20.

9. Cole TJ, Bellizzi MC, Flegal KM, Dietz WH. Establishing a standard definition for child overweight and obesity worldwide: international survey. BM] 2000;320:1240-3.

10. Rexrode KM, Buring JE, Manson JE. Abdominal and total adiposity and risk of coronary heart disease in men. Int J Obes Relat Metab Disord 2001;25:1047-56.

11. Wei M, Gaskill SP, Haffner SM, Stern MP. Waist circumference as the best predictor of noninsulin dependent diabetes mellitus (NIDDM) compared to body mass index, waist/hip ratio and other anthropometric measurements in Mexican Americans-a 7-year prospective study. Obes Res 1997;5:16-23.

12. Amato MC, Giordano C, Galia M, et al.; AlkaMeSy Study Group. Visceral Adiposity Index: a reliable indicator of visceral fat function associated with cardiometabolic risk. Diabetes Care 2010;33:920-2.

13. Amato MC, Giordano C, Pitrone M, Galluzzo A. Cut-off points of the visceral adiposity index (VAI) identifying a visceral adipose dysfunction associated with cardiometabolic risk in a Caucasian Sicilian population. Lipids Health Dis 2011;10:183.

14. Bozorgmanesh M, Hadaegh F, Azizi F. Predictive performance of the visceral adiposity index for a visceral adiposity-related risk: type 2 diabetes. Lipids Health Dis 2011;10:88.

15. Mohammadreza B, Farzad H, Davoud K, Fereidoun Prof AF. Prognostic significance of the complex "Visceral Adiposity Index" vs. simple anthropometric measures: Tehran lipid and glucose study. Cardiovasc Diabetol 2012;11:20.

16. Amato MC, Verghi M, Galluzzo A, Giordano C. The oligomenorrhoic phenotypes of polycystic ovary syndrome are characterized by a high visceral adiposity index: a likely condition of cardiometabolic risk. Hum Reprod 2011;26:1486-94.

17. Oh JY, Sung YA, Lee HJ. The visceral adiposity index as a predictor of insulin resistance in young women with polycystic ovary syndrome. Obesity 2012 21:1690-4.

18. Amato MC, Giordano C. Clinical indications and proper use of Visceral Adiposity Index. Nutr Metab Cardiovasc Dis 2013;23:e31-2.
19. Al Herbish AS, El Mouzan MI, Al Salloum AA, et al. Body mass index in Saudi Arabian children and adolescents: a national reference and comparison with international standards. Ann Saudi Med 2009;29:342-7.

20. Petta $S$, Amato M, Cabibi D, et al. Visceral adiposity index is associated with histological findings and high viral load in patients with chronic hepatitis $C$ due to genotype 1. Hepatology 2010;52:1543-52.

21. Ciresi A, Amato MC, Pizzolanti G, Giordano Galluzzo C. Visceral adiposity index is associated with insulin sensitivity and adipocytokine levels in newly diagnosed acromegalic patients. J Clin Endocrinol Metab 2012;97:2907-15.

22. Al-Daghri NM, Al-Attas OS, Alokail MS, et al. Visceral adiposity index is highly associated with adiponectin values and glycaemic disturbances. Eur J Clin Invest 2013;43:183-9.

23. Björntorp P. [Metabolic difference between visceral fat and subcutaneous abdominal fat]. Diabetes Metab 2000;26:Suppl 3:10-2.

24. Falaschetti E, Hingorani AD, Jones A, et al. Adiposity and cardiovascular risk factors in a large contemporary population of pre-pubertal children. Eur Heart J 2010;31:3063-72.

25. Xekouki P, Nikolakopoulou NM, Papageorgiou A, et al. Glucose dysregulation in obese children: predictive, risk, and potential protective factors. Obesity (Silver Spring) 2007;15:860-9.

26. Kanaka-Gantenbein C, Margeli A, Pervanidou P, et al. Retinol-binding protein 4 and lipocalin-2 in childhood and adolescent obesity: when children are not just "small adults". Clin Chem 2008;54:1176-82.

27. Maffeis C, Manfredi R, Trombetta M, et al. Insulin sensitivity is correlated with subcutaneous but not visceral body fat in overweight and obese prepubertal children. J Clin Endocrinol Metab 2008;93:2122-8.

28. Murdolo G, Nowotny B, Celi F, et al. Inflammatory adipokines, high molecular weight adiponectin, and insulin resistance: a population-based survey in prepubertal schoolchildren. PLoS ONE 2011;6:e17264.

29. Al-Daghri NM, Al-Attas OS, Alokail MS, et al. Parent-offspring transmission of adipocytokine levels and their associations with metabolic traits. PLoS ONE 2011;6:e18182. 